University of Wollongong

Research Online

$1-1-2019$

\title{
The Paediatric electronic Persistent Pain Outcomes Collaboration (PaedePPOC): establishment of a binational system for benchmarking children's persistent pain services
}

\author{
Susan Lord \\ Hunter Medical Research Institute, John Hunter Children's Hospital, University of Newcastle \\ Hilarie Tardif \\ University of Wollongong, hilarie@uow.edu.au \\ Elizabeth A. Kepreotes \\ University of Newcastle, John Hunter Children's Hospital, Hunter Medical Research Institute \\ Megan B. Blanchard \\ University of Wollongong, mblancha@uow.edu.au \\ Kathy Eagar \\ University of Wollongong, keagar@uow.edu.au
}

Follow this and additional works at: https://ro.uow.edu.au/ahsri

Research Online is the open access institutional repository for the University of Wollongong. For further information contact the UOW Library: research-pubs@uow.edu.au 


\title{
The Paediatric electronic Persistent Pain Outcomes Collaboration (PaedePPOC): establishment of a binational system for benchmarking children's persistent pain services
}

\begin{abstract}
Chronic or persistent pain is a growing global health problem. Effective management of pain emerging in childhood may prevent long-term health and vocational consequences. Internationally, paediatric pain services are a limited resource and, as such, must strive to improve equity, outcomes, and value for money. The Paediatric electronic Persistent Pain Outcomes Collaboration (PaedePPOC) is a binational paediatric outcome measurement centre that aims to measure, benchmark, and improve children's specialist pain services in Australasia. This study documents the establishment of PaedePPOC and presents baseline and initial outcome data. Binational consensus meetings determined the measures. Governance structures, collection protocols, information technology, site-specific logistics, and onsite training were achieved within 18 months. Children and parents complete baseline and progress questionnaires. Seven of 10 Australasian services provided data to PaedePPOC, with 1432 patients enrolled until June 2018. At baseline, patients were $12.4 \pm$ (3.0) years, $68 \%$ female, $93 \%$ Australian-born, and $5 \%$ Aboriginal and/or Torres Strait Islander people. Most had moderate-severe functional disability and impaired quality of life, with pain affecting school attendance and employment. Opioid-containing medicines were used often or daily by $16 \%$. Patients completing outcome measures at treatment end reported clinically significant improvement in pain intensity (49\% of patients), functional ability (59\%), and quality of life (69\%). The PaedePPOC initiative has been successfully integrated into children's pain services, yielding timely point-of-care information to support clinicians and families, and valuable binational and service data to inform quality improvement and future sector planning.

\section{Publication Details}

S. Lord, H. P. Tardif, E. A. Kepreotes, M. Blanchard \& K. Eagar, "The Paediatric electronic Persistent Pain Outcomes Collaboration (PaedePPOC): establishment of a binational system for benchmarking children's persistent pain services", Pain 1607 (2019) 1572-1585.
\end{abstract}

This journal article is available at Research Online: https://ro.uow.edu.au/ahsri/972 


\section{PaedePPOC - the Paediatric Electronic Persistent Pain Outcomes \\ Collaboration: Establishment of a bi-national system for benchmarking children's persistent pain services}

\section{Authors}

Susan M Lord PhD ${ }^{1,2,3}$, Hilarie $\mathrm{P}$ Tardif $\mathrm{PhD}^{4}$, Elizabeth $\mathrm{A}$ Kepreotes $\mathrm{PhD}^{1,3,6,7}$, Megan Blanchard BMath ${ }^{4}$ and Kathy Eagar PhD ${ }^{5}$

\section{Affiliations}

${ }^{1}$ Children's Complex Pain Service, John Hunter Children's Hospital, Newcastle, Australia

${ }^{2}$ School of Medicine and Public Health, University of Newcastle, Callaghan, Australia

${ }^{3}$ Hunter Medical Research Institute, Newcastle, Australia

${ }^{4}$ electronic Persistent Pain Outcomes Collaboration, Faculty of Business, University of Wollongong, Wollongong, Australia

${ }^{5}$ Australian Health Services Research Institute, Faculty of Business, University of Wollongong, Wollongong, Australia

${ }^{6}$ Priority Research Centre GrowUpWell ${ }^{\circledR}$ University of Newcastle, Callaghan, Australia

${ }^{7}$ School of Nursing and Midwifery, University of Newcastle, Callaghan, Australia

Number of Text Pages 43 (including track changes, references and figure legends)

Number of Figures $\quad 2$

Number of Tables $\quad 6$

\section{Correspondence}

Hilarie Tardif, PhD, Australian Health Services Research Institute, iC Enterprise 1, Innovation Campus, University of Wollongong, NSW 2522, Australia. Tel: +61-2-4221-4023; FAX: +61-2-4221-4679; Email: hilarie@uow.edu.au 


\section{Disclosure}

No authors have any conflicts of interest to report.

\section{Previous presentations}

The abstract and manuscript have not been published or presented previously. Aspects of the research - the evolution of the dataset, and earlier data releases-have been included in conference presentations:

Lord SM. Measuring Outcomes of Children's Complex Pain Services. Australian Pain Society ASM, Hobart Australia 14/04/2014.

Lord SM, Kepreotes EA. Implementing PaedePPOC. ePPOC Forum, Sydney Australia 17/09/2015.

Lord SM, Tardif H. ePPOC - the electronic persistent pain outcomes collaboration. Topical Session. International Symposium on Pediatric Pain, Seattle Washington USA 02/06/2015.

Blyth F, Tardif H, Lord SM. ePPOC across epochs. Topical session. Australian Pain Society ASM, Adelaide Australia 10/04/2017.

Some baseline data were presented in an online Information Series for participating services:

H Tardif, M Blanchard, M Bryce \& J White, 'Normative data for children and adolescents referred for specialist pain management in Australia', ePPOC Information Series No. 2, 2018.

https://ahsri.uow.edu.au/content/groups/public/@web/@chsd/documents/doc/uow251871.pdf 
SUMMARY [22 words]

PaedePPOC has been successfully established and embedded in Australian children's persistent pain services, laying the foundation for sector-wide benchmarking and quality improvement.

\section{KEY WORDS}

Paediatric / pediatric, child, persistent pain, outcomes, benchmark, registry

\section{ACRONYMS}

\begin{tabular}{|l|l|}
\hline AHSRI & Australian Health Services Research Institute \\
\hline BAPQ & Bath Adolescent Pain Questionnaire \\
\hline BAP-PIQ & Bath Adolescent Pain Parent Impact Questionnaire \\
\hline BPI-Mod & Modified Brief Pain Inventory \\
\hline ePPOC & electronic Persistent Pain Outcomes Collaboration \\
\hline FDI & Functional Disability Inventory \\
\hline NPS & National Pain Strategy \\
\hline PaedePPOC & Paediatric electronic Persistent Pain Outcomes Collaboration \\
\hline Parent & $\begin{array}{l}\text { Adult legal guardian and carer for a child, whether the } \\
\text { biological parent or in loco parentis }\end{array}$ \\
\hline PedsQL & Pediatric Quality of Life \\
\hline PRG & Paediatric Reference Group \\
\hline PROMs & Patient-Reported Outcome Measures \\
\hline
\end{tabular}




\section{ABSTRACT [236/250 words]}

Chronic or persistent pain is a growing global health problem. Effective management of pain emerging in childhood may prevent long-term health and vocational consequences. Internationally, paediatric pain services are a limited resource and, as such, must strive to improve equity, outcomes and value for money. The Paediatric electronic Persistent Pain Outcomes Collaboration (PaedePPOC) is a bi-national paediatric outcome measurement centre that aims to measure, benchmark, and improve children's specialist pain services in Australasia. This study documents the establishment of PaedePPOC and presents baseline and initial outcome data. Bi-national consensus meetings determined the measures. Governance structures, collection protocols, information technology, sitespecific logistics and onsite training were achieved within 18 months. Children and parents complete baseline and progress questionnaires. Seven of ten Australasian services provided data to PaedePPOC, with 1432 patients enrolled to June 2018. At baseline, patients were $12.4 \pm[3.0]$ years, $68 \%$ female, 93\% Australian-born, and 5\% Aboriginal and/or Torres Strait Islander people. Most had moderate-severe functional disability and impaired quality of life, with pain affecting school attendance and employment. Opioid-containing medicines were used often or daily by $16 \%$. Patients completing outcome measures at treatment end reported clinically significant improvement in pain intensity (49\% of patients), functional ability (59\%) and quality of life (69\%). The PaedePPOC initiative has been successfully integrated into children's pain services, yielding timely point-of-care information to support clinicians and families, and valuable bi-national and service data to inform quality improvement and future sector planning. 


\section{INTRODUCTION [455/500 words]}

Chronic or persistent pain is recognised as a significant public health problem globally, ${ }^{10,21,30,45,55}$ and affects almost one in five Australians ${ }^{9,39}$ and New Zealanders. ${ }^{20}$ It is associated with high costs, not just in terms of healthcare expenditure, disability payments, and prescription-analgesic morbidity and mortality, but also due to lost employment, productivity and social capital. ${ }^{1,11,34,45}$

Persistent pain in childhood and adolescence is similarly common ${ }^{50,60,64}$ and costly. ${ }^{12,37,42,63}$ If inadequately treated, childhood pain can interrupt physical, cognitive, emotional and social development. ${ }^{19,23,46,61}$ The resultant disability and comorbidities may continue into adulthood, ${ }^{2,40,74}$ with long-term consequences and costs for the individual and society.

In Australia and New Zealand, some 5-19\% of adolescents and young people experience intrusive persistent pain. ${ }^{9,20,39,60}$ However, children and adolescents who require care for persistent pain remain relatively underserviced, with estimates that new assessments by specialist pain services are made for less than $1 \%$ of young Australians..$^{43}$ In 2018, children's services remain few and far between, with only nine services across Australia and one in New Zealand.

With such a small sector to support the needs of so many children with persistent pain, it is imperative that children's specialist pain services develop means to measure patient outcomes to improve the quality, effectiveness, and value of care. Rigorous clinical trials and systematic reviews identify treatments that ought to be best practice, but few include children with persistent pain. ${ }^{15-}$ 18,24,25,27,31 Furthermore, clinical trial patients and conditions often vary markedly from real-world clinical contexts. Measures of efficacy need to be followed by measures of effectiveness to improve generalisability.

For many conditions and healthcare interventions, outcome registries are showing great potential to improve the quality and value of care. ${ }^{44,51,76}$ By identifying variations in outcomes, registries allow development of benchmarks and assessment of comparative performance at the clinic, regional, national, and international levels. ${ }^{51}$ Examining and learning from the practices of high-performing 
services, through a continuous quality improvement process, may lead to earlier identification and dissemination of best practices.

Recognising this, in 2012, the Australian and New Zealand children's persistent pain management sector began a collaborative process to identify a minimum set of outcome domains and measures with which to measure and compare service effectiveness and value. Parallel endeavours in the adult persistent pain sectors in Australia, ${ }^{63}$ Canada, ${ }^{13}$ the UK, ${ }^{57}$ and USA ${ }^{52}$ have been described. To date, USA is the only other country to report the establishment of an outcomes registry for children's specialist pain services (Peds-CHOIR). ${ }^{7}$ A registry has been initiated in Ontario, Canada ${ }^{12}$ but is yet to be reported.

This paper describes the political and logistic processes whereby the Australian and New Zealand electronic Persistent Pain Outcomes Collaboration for children's specialist pain services (PaedePPOC) was established, and outlines the methods and resources this required. Baseline and initial outcome data for children are reported. 


\section{METHOD}

\section{History of PaedePPOC}

In 2005, the Faculty of Pain Medicine of the Australian \& New Zealand College of Anaesthetists (FPM ANZCA) recognised that a national outcomes dataset would facilitate benchmarking and quality improvement across the specialty. The Faculty established a working group to garner clinician support for an outcomes collaboration and to identify preliminary consensus on key outcome domains. In 2010, this group, along with other professional bodies and consumer stakeholders, participated in a National Pain Summit that yielded the National Pain Strategy (NPS). ${ }^{58}$ One of the NPS priority objectives was quality improvement through outcomes measurement, reporting, and national benchmarking. The NPS also recognised the importance of developing programs to specifically evaluate persistent pain management in children and adolescents. ${ }^{58}$

The ability to realise this important objective came in 2012 with the announcement that the New South Wales (NSW) Ministry of Health would fund the establishment of an outcomes database for specialist pain management services. This followed considerable planning and advocacy by the NSW Agency for Clinical Innovation (ACl) Pain Management Network and a Ministerial Taskforce. ${ }^{59}$ The resultant initiative, named the electronic Persistent Pain Outcomes Collaboration (ePPOC) would ensure consistent and standardised data collection and reporting in NSW services, and across Australia and New Zealand. ${ }^{65}$

Throughout this evolution, members of the paediatric service sector were involved in the various professional bodies and meetings, advocating for children and their families. Advocacy efforts were facilitated by the formation of a Pain in Childhood Special Interest Group within the Australian and New Zealand Pain Societies in $2011 .{ }^{4}$ Consequently, ePPOC became an initiative of the pain management sector to improve outcomes for all patients experiencing persistent pain, irrespective of their age. 
Implementation of ePPOC began in 2013 when the Australian Health Services Research Institute (AHSRI), based at the University of Wollongong, was chosen to develop and manage the ePPOC and PaedePPOC projects. AHSRI had established similar outcome collaborations in the related fields of rehabilitation (AROC, the Australasian Rehabilitation Outcomes Centre) and palliative care (PCOC, the Palliative Care Outcomes Collaboration) and had experience in implementing national patient outcome measurement suites and reports for benchmarking, research, and governance. This was, however, the first time specialist children's services had been involved alongside adults' services from the inception of such a collaboration. Whilst there is much in common, important differences between adult and children's service models, client needs, and validated outcome measures necessitated a parallel but separate development and reporting process.

\section{Governance}

During establishment, a National Reference Group was formed comprising governmental and clinical representatives from the Australian states and territories and New Zealand. This group refined the ePPOC minimum dataset for adults' services, and developed the protocol for data collection and implementation resources. A Paediatric Reference Group (PRG) supervised the parallel development of the paediatric dataset and collection protocol for children's services (PaedePPOC). Following the implementation phase of ePPOC and PaedePPOC, a Management Advisory Group and a Scientific and Clinical Advisory Committee were formed to oversee and support the continued development of the outcome registries. ${ }^{28}$ These advisory groups have recently been amalgamated to form a singular Clinical and Management Advisory Committee (CMAC). Paediatric representation continues within this framework.

Implementation and the day-to-day operations of PaedePPOC are managed by AHSRI. The staff currently employed to support ePPOC and PaedePPOC are a director, administrator and statistician, and two project coordinators whose roles are to assist services to collect, interpret and use their data for quality improvement purposes. 


\section{Ethics and Privacy}

The establishment of ePPOC and PaedePPOC were approved by the University of Wollongong Human Research Ethics Committee. This approval allows non-identified patient data to be collected from participating pain services for the purposes of reporting, quality assurance, benchmarking and research. EPPOC and PaedePPOC were deliberately established as patient non-identified to allow collection of information from the pain services without the requirement for explicit patient consent for this information to be forwarded. This was done to ensure that the database is complete and representative, and that the resulting reports, quality improvement initiatives, benchmarks and research are meaningful and accurate.

Individual services own their data and are responsible for the privacy and ethical use of identified and re-identifiable healthcare information stored within their own systems. Every six months, each participating service sends a patient non-identified data extract to PaedePPOC via a secure upload facility. Although non-identifiable once uploaded, the PaedePPOC data contains a statistical linkage key ${ }^{56}$ computed automatically from mandatory fields by the software at the time of data entry. This enables data from multiple episodes of care at one centre, or multiple centres across childhood and adulthood, to be linked longitudinally without identifying the source.

Services receive confidential reports of their own facility-level data alongside the pooled data for all services, whereas public reports contain only pooled data. This service-level anonymity is critical to the success of the collaboration as it helps to remove concerns that there may be external incentives or ramifications for achieving, or not achieving, particular outcomes.

The PaedePPOC data stored at the University of Wollongong is available to be used for approved research projects by participating pain management services and external researchers. A Data Access Working Group is responsible for reviewing and approving applications for data and providing advice to applicants. All research projects using ePPOC and PaedePPOC data must obtain separate ethics approval. 


\section{PaedePPOC Minimum Dataset}

During 2013, the PRG followed closely the progress and decisions in the development of the adult minimum dataset. It was considered important to maintain the same core outcome domains across the lifespan, whilst recognising the need to employ developmentally appropriate and age-validated measurement tools within each domain. In order to arrive at agreement on the domains and measures, members of the PRG performed a pragmatic literature review, conducted an email discussion group, and attended two face-to-face consensus meetings.

A number of guiding principles were followed in determining the minimum dataset. Of critical importance were the priorities of ensuring maximal clinical utility whilst minimising the child/family data collection burden. Accordingly, preference was given to measures that were easy, brief, free to use, validated for relevant ages and contexts, sensitive to change, and facilitated clinical processes such as triage, assessment and monitoring of progress. In addition, it was agreed that PROMs should be preeminent. Parent-proxy measures would also be collected where possible to allow assessment of children too young or unable to complete the patient-rated tools, and to allow investigation of child-parent dyads.

All measures had to serve at least one of the following functions:

- $\mathbf{P}$ - Patient or problem descriptors i.e. pain, comorbidity, disability;

- $\mathbf{E}$ - Exposure descriptors i.e. specialist pain service type and timing;

- $\quad$-Outcomes (child and/or parent reported).

\subsection{Domains}

The PRG considered the domains recommended for the adult minimum dataset together with the PedIMMPACT Consensus outcome domains developed for reporting paediatric persistent pain clinical trials. ${ }^{53}$ The final list of outcome domains for children's services (Table 1 ) is a composite of the two sources with some omissions and one addition. Omitted were the PedIMMPACT domains 'Treatment-emergent adverse symptoms/events' and 'Global judgement of satisfaction with 
treatment' due to lack of validated patient-reported measures and potential bias in the absence of an independent assessor. 'Economic factors' was omitted due to the burden of collecting reliable data. It was agreed that consideration of these domains be revisited for subsequent versions. The PRG included the impact of the child's pain on their parents, as this was considered an important baseline, progress and outcome domain with immediate clinical relevance.

Table 1 here

\subsection{Data items}

\subsubsection{Patient and Problem Measures}

Baseline demographics include age, sex, height and weight, school stage, employment, residential postcode, compensation status, Indigenous status and country of birth. The child's comorbidities (chronic disease, mental health, cancer) and disabilities (sight or hearing impairment, intellectual or physical disability) are also recorded by the parent. Pain problem descriptors include parentreported cause at onset and how long the pain has been experienced. Both parent and child report the frequency and location of the pain. The latter is recorded as the single worst location, and all other locations of pain on a body-map. ${ }^{72}$

\subsubsection{Exposure Measures}

Recognising that there is considerable variation in treatment between services, the collaboration seeks to capture service activity data in order to determine which types and intensities of treatment were associated with better outcomes. There is also interest in evaluating best value for outcome. Wait-time is calculated as the number of days from receipt of referral by the service to commencing an episode of care. An episode of care is defined as a continuous period of care for a patient within one pain management service. Episode start and end dates, referral source and disposition at discharge are recorded. Within an episode, patients may receive different types of care usually predicated by their progress. These different types of care are categorised into pathways (e.g. 
individual appointments, group program, procedural intervention) to enable comparative evaluation of the outcomes of different pathways in addition to overall episode outcome. Within an episode of care, each service event date, duration, and type is recorded. Remotely-delivered events by telephone and telehealth are also captured.

\subsubsection{Outcome Measures (PROMs)}

The outcome measures chosen to assess each domain were determined by evidence-informed and pragmatic consensus. Along with the guiding principles outlined previously, the specific materials considered included: measures in current use by member services; individual measures recommended by the PedIMMPACT Consensus, ${ }^{53}$ and a later FDA Scientific Workshop on paediatric analgesic trial design and measures; ${ }^{6}$ an unpublished literature review conducted by a member service (Pain Research Unit, Sydney Children's Hospital Randwick Discussion Document (unpublished) 2011); together with recently developed and emerging measures. Furthermore, the PRG explored the progress of similar initiatives in other nations with a view to the possibility of aligning in the interests of future international benchmarking and research.

Three child-report questionnaire batteries were developed to accommodate different developmental levels $-5-7$ years, 8-12 years and 13-18 years-requiring different age-valid measures. A separate battery for patients aged under 5 years was considered, however due to the small number typically presenting to services, it was decided that specific measures for this age group would not be included. Children aged 5-7 years, with assistance from a clinician or parent, complete 28 questionnaire items; 8-12 year olds complete 44 items; and 13-18 year old adolescents complete 51 items. Parents of children 0-18 years answer 61 items relating to their child and 62 items relating to themselves at referral. Subsequent questionnaires are of equal length except that parents only need to complete 52 items relating to their child. Individual services can weigh up the benefit and burden of collecting additional measures for local use or research. Most participants choose to complete questionnaires online, and parents typically report that the child and parent 
questionnaires each take approximately ten minutes to complete. However, if the questionnaires are delivered verbally (due to poor literacy for example), completion may take around 20 minutes. These timeframes are comparable to those reported for the Peds-CHOIR assessment measures. ${ }^{7}$

Table 2 lists the outcome domains and assessment tools that constitute the core minimum dataset. Each of the standardised assessment tools are described in more detail below.

Table 2 here

Body Map

The body map developed by the Childhood Arthritis and Rheumatology Research Alliance (CARRA) $)^{72}$ is used to record the location of the main and additional pain. The delineated body regions in this map make online selection of areas easier, and also facilitates standardised reporting of pain location.

\section{Modified Brief Pain Inventory}

The Brief Pain Inventory (BPI) ${ }^{14}$ is a measure assessing pain intensity and pain interference with daily activities. PaedePPOC assesses pain intensity in children aged eight and over using questions based on the four pain intensity questions in the BPI, but with slight modification to ensure they are appropriate for children experiencing chronic pain. Four questions ask the child to rate their pain intensity at its usual (instead of "average" ${ }^{14}$ ), worst, and least in the last week, and their pain intensity now. Pain intensity is rated on a 0 to 10 numeric rating scale where 0 represents "no pain", and 10 represents "pain as bad as you can imagine" in the adolescent questionnaires and "worst pain ever" for children aged 8-12 years. Parents complete this assessment tool regardless of the age of their child. Children and parents are asked to rate the pain intensity over the past week, consistent with recommendations for a longer assessment period in chronic pain. ${ }^{53}$ Similar questions are included in Peds-CHOIR, with pain reported over the past month. ${ }^{4}$ Pain intensity is reported by PaedePPOC as mild (0-4), moderate (5-6) or severe (7-10). An improvement of $30 \%$ or more from baseline has been recommended as representing moderate clinically significant improvement for 
adult studies of chronic pain. ${ }^{22}$ This measure of improvement is used in ePPOC for the adult population and was also adopted in PaedePPOC for consistency.

While the BPI is not commonly used in paediatric studies, PaedePPOC chose to include pain intensity questions based on those in the BPI due to ease of use and scoring, and to be consistent with the questions asked in ePPOC. In addition, studies have shown numeric pain rating scales similar to those used in PaedePPOC to be valid and psychometrically sound in paediatric patients. ${ }^{8,54,73}$

\section{Faces of Pain Scale-Revised}

Children aged five to seven complete the Faces of Pain Scale - Revised (FPS-R $)^{41}$ to report the severity of their pain. This assessment tool presents six faces showing increasing levels of pain. The clinician or parent reads standardised instructions, which include asking the child to indicate which face reflects the pain the child feels. The six faces are scored $0,2,4,6,8$ and 10 , with 0 representing "no pain" and 10 "very much pain". The FPS-R is freely available through the International Association for the Study of Pain. It is widely used and available in many languages. Consistent with the pain severity questions described for the older children, a $30 \%$ or greater improvement is considered clinically significant for the purposes of reporting in PaedePPOC.

\section{Functional Disability Inventory}

The Functional Disability Inventory $(\mathrm{FDI})^{75}$ is a tool used to assess functional limitation in children. Fifteen items ask patients whether they have had any physical trouble or difficulty doing specified activities over the past two weeks. Items are rated on a five point scale where $0=$ No trouble, $1=\mathrm{A}$ little trouble, 2=Some trouble, 3=A lot of trouble and 4=Impossible. A total score is calculated by summing responses on each item, giving a range of 0-60 with higher scores reflecting higher levels of disability. The FDI is a widely used assessment tool and has been recommended as a functional outcome measure in studies of paediatric chronic pain. ${ }^{53}$ Severity bands for the FDI are no/minimal disability (0-12), moderate disability (13-29) and severe disability $(>29) \cdot{ }^{49}$ At inception we were unable to find published recommendations regarding clinically meaningful change on this measure, 
so the PRG suggested that for the purposes of PaedePPOC, this should be defined as a change of five or more points coupled with a change to a different severity category. Subsequently, another group published a more rigorous recommendation for reliable clinical change being $\geq 7.8$ point reduction coupled with a change in FDI severity category. ${ }^{62}$ Nevertheless, to enable longitudinal service evaluation, PaedePPOC currently continues to use its original definition of clinically meaningful change.

Pediatric Quality of Life Inventory

PaedePPOC uses the Pediatric Quality of Life (PedsQL) ${ }^{67}$ Generic Core Scales to measure healthrelated quality of life. Parents and children complete the age-appropriate version. The PedsQL assesses physical, emotional, social and school functioning, and asks how much of a problem the child has had with each of these over the past week. The tool consists of 23 questions each rated on a five point scale where $0=$ Never [a problem], 1=Almost never, $2=$ Sometimes, $3=$ Often and $4=$ Almost always. For children aged 5-7 years the scale is clinician-administered and rated on a three point scale where $0=$ Never [a problem], 2=Sometimes and 4=Almost always.

All PedsQL item responses are reverse scored and transformed to a 0-100 scale, with higher scores indicating better health-related quality of life. Results are reported for the total score, each of the subscales (physical, emotional, social and school functioning) and two summary scores reflecting psychosocial and physical health. PaedePPOC also reports the single item assessing sleep.

The PedsQL is widely used and recommended as a tool to measure parent and child report of physical, emotional, school and social functioning in studies of paediatric chronic pain. ${ }^{53}$ Minimal clinically meaningful difference is measured as a 4.4 point change in the child self-report total score, and a 4.5 point change in the adult proxy-report total score. ${ }^{67}$

Bath Adolescent Pain Questionnaire (BAPQ) - Pain Specific Anxiety subscale

The BAPQ is a 61 item measure which assesses the impact of pain over seven domains: social functioning, physical functioning, depression, general anxiety, pain specific anxiety, family 
functioning and development. ${ }^{26}$ The BAPQ has been specifically developed for use with adolescents experiencing chronic pain and has been shown to be a reliable and valid measure in this population. ${ }^{26,33}$ While the entire assessment tool was considered for inclusion in the PaedePPOC minimum dataset, the preference was for measures that could span multiple age groups, such as the FDI and PedsQL. Given the importance of assessing pain-related cognitions however, the PRG decided to include the seven-item pain-specific anxiety subscale of the BAPQ for adolescent patients to complete.

This subscale assesses worries or concerns the patient has about their pain. Patients are asked to read a list of statements and mark the answer that best describes how often they have experienced each in the past two weeks. Items are rated using a five point scale, where $0=$ Never, $1=$ Hardly ever, $2=$ Sometimes, $3=$ Often and 4=Always. Item scores are totalled with a range of 0 to 28 . Higher scores indicate higher levels of pain-specific anxiety. Severity bands and guidelines for clinically significant change on this measure have not yet been established.

\section{Bath Adolescent Pain Parent Impact Questionnaire (BAP-PIQ)}

The BAP-PIQ has been specifically developed to assess the impact of a child's pain on the life of the parent and has been shown to be a reliable and valid measure. ${ }^{47}$ The questionnaire consists of eight subscales addressing depression (9 items), anxiety (6 items), child-related catastrophizing (5 items), self-blame and helplessness ( 7 items), partner relationship (7 items), leisure functioning (8 items), parental behaviour (11 items) and parental strain ( 9 items). Parents are asked to read each statement and indicate how often each was experienced in the last two weeks. Items are rated using a five point scale, where $0=$ Never, $1=$ Hardly ever, $2=$ Sometimes, $3=$ Often and $4=$ Always. Scores are reported for each subscale, with higher scores reflecting greater impairment. The current paper focusses on child baseline and outcome data however, and scores on the BAP-PIQ will not be reported here. 
It should be noted that the PRG agreed to these domains, data items and assessment tools with the expectation that the minimum dataset could be updated if required. A process allowing for continuous review and evaluation was therefore implemented, whereby participating services and stakeholders can submit requests to PaedePPOC justifying a change or addition to the data collection. These suggestions are then discussed among the PRG and also reviewed at CMAC meetings. For logistical reasons and to maintain longitudinal information, release of a new dataset is only expected every few years. To date, there has been one version update, incorporating minor changes to data items and code sets rather than assessment tools. However, planning is underway to implement more significant changes in a future version, including introduction of a specific measure to assess sleep, and an item enabling collection of pain diagnoses. As has occurred in ePPOC, candidate measures and data items will first be trialled and evaluated in a select number of pain management units before a decision is made to incorporate into the PaedePPOC software and minimum dataset.

\section{Participants}

All specialist paediatric pain management services in Australia and New Zealand are eligible to participate in PaedePPOC. To date, seven of ten Australasian paediatric services have implemented PaedePPOC at their service. Another two services have recently joined and will begin to submit data in 2019.

\section{Data collection and quality}

The purpose-built software (epiCentre), protocol for collecting data, Data Policy and Data Dictionary are described in detail elsewhere. ${ }^{65}$ In brief however, questionnaires are generated electronically when a referral is logged in epiCentre. Depending on child/parent preference they can be emailed and completed online, posted, or completed in clinic either on paper or on a tablet. The subsequent primary collection points are at the end of the episode of care, and 3-6 months after the episode has 
ended. Questionnaires can also be collected throughout the episode of care to review progress, as deemed appropriate by individual pain services.

epiCentre facilitates data checking and error minimisation by incorporating a number of validation checks at point-of-entry. These include mandatory fields (e.g. sex, date of birth, questionnaire completion dates) and logic checks (e.g. an episode of care beginning before a referral has been received). Following each data submission, PaedePPOC produces a short report summarizing the data provided and identifying omissions or improbable data. This information is discussed with each pain management service, with services encouraged to correct their data and resubmit.

\section{Reporting}

Individual patient scores and progress reports are generated electronically by pain service staff using epiCentre. These individual reports enable documentation at the clinic level, and facilitate discussion with the individual child, family and referrer. Once a child and family have completed more than one set of PaedePPOC questionnaires, the report generated will graphically compare their most recent scores with baseline scores (see example in Figure 1). This enables children as developmentallyappropriate, families, and referrers to visualise quantitatively the changes that they may have observed qualitatively.

Figure 1 here

Each participating pain management service receives a detailed report every six months (JanuaryDecember and July to June), comparing the service's data along-side aggregated data from all participating services. Reports contain a descriptive profile of patients seen during the reporting window, the service activity profile and service outcomes. The proportion of patients who achieve pre-defined clinically significant change from baseline is also reported. An online example report is publicly available at https://ahsri.uow.edu.au/eppoc/reports/. ${ }^{29}$ Each pain service report is relayed in confidence to the service's nominated contact person and is not released or discussed externally 
by PaedePPOC. Individual services may elect to discuss their own data locally, or in benchmarking or scientific meetings.

EPPOC and PaedePPOC also release an annual report which describes, at a composite level, the patients referred to adult and paediatric pain units in Australasia, along with the services they received and the outcomes achieved. ${ }^{29}$ As the national and service-level reports describe patients, services and outcomes over a discrete period, they may be used to evaluate the impact of changes in health care delivery. For example, a pain service interested in trialling a new form of pain management can use a current report as a baseline, with future reports reflecting outcomes as a result of the new treatment. 


\section{RESULTS}

\section{Implementation and challenges}

PaedePPOC was established in 2013 and implemented in paediatric pain management services from 2014. As at January 2019, nine of the ten specialist paediatric pain management services in Australia and New Zealand had joined the collaboration.

Many of the challenges faced during this implementation period mirrored those experienced during implementation of ePPOC, the collaboration for adult pain services. As described previously, ${ }^{65}$ one of the least expected hurdles was the difficulty installing the software program epiCentre at each pain management service. epiCentre is a small program, however obtaining support and security clearance from information technology (IT) departments within hospitals was time consuming and meant delays in joining the collaboration for many services. Periodic updates to the software also require hospital IT involvement, and while the installation of these is less onerous, establishing an ongoing relationship with each hospital's IT staff has become a necessary function for ePPOC and PaedePPOC.

Another challenge identified in the implementation phase of both ePPOC and PaedePPOC was to embed the standardised protocol and terminology into the unique and often well-established processes and practice of each pain management service. This is an important issue because ensuring that questionnaires are collected at consistent points in and after an episode is crucial to obtaining meaningful and comparable data. ePPOC staff attempted to facilitate this process by firstly visiting each new service and together developing a 'process map'. This outlines the typical patient pathway through the pain management service and highlights when and how to collect the questionnaires, and when to enter data into epiCentre. This education and training has become an important ongoing function for ePPOC staff, involving training of new pain service staff, checking that submitted data complies with the protocol, and assisting pain services to understand and use their reports. 
An ongoing hurdle for many services is the data entry requirements of PaedePPOC participation.

epiCentre is a stand-alone program, and there is therefore some double entry of data (e.g. name, date of birth, referral date) into epiCentre and the hospital's medical record system. There is no easy solution to this due to the variety of hospital systems used in each jurisdiction, and limited IT resources within EPPOC and the individual hospitals. In recognition of this additional burden for pain service staff however, ePPOC has attempted to promote use of epiCentre through continuously improving useability of the software and highlighting the clinical benefits of point-of-care data and tracking of patients.

Despite these challenges, seven of Australasia's ten specialist paediatric pain services had joined the collaboration and provided data during the period January 2014 to June 2018. These services were all located in Australia, in the states of Queensland, NSW and Victoria, and based in major cities. All services are tertiary referral pain services in a hospital setting. Six services are publically funded and one is a private service. Pain services joined PaedePPOC at different times throughout this period, with the average participation period being 36 months (range 20 to 50).

The services submitted data for 1432 children. Questionnaires were completed online (80.9\%), in clinic (15.6\%) and via post (3.5\%). Within returned questionnaires, over $95 \%$ of all assessment tools were validly completed, suggesting patient and parent acceptability of the measures.

Referral questionnaires were completed by 1076 children and 1108 parents. Approximately one in four patients ( $n=356,24.9 \%)$ and parents $(n=324,22.6 \%)$ had not completed a referral questionnaire at the time of data extraction. The reasons for this include that they were referred and registered in epiCentre but did not engage further with the pain service, they had not yet completed or been sent a questionnaire at the time the data were submitted to PaedePPOC, or they were unable to complete a questionnaire. Of the parents who reported that their child was unable to complete their questionnaire $(n=102), 96$ reported a reason. These were refusal $(4.2 \%)$, that their child was too young $(41.7 \%)$, cognitively unable $(17.7 \%)$, physically unable $(8.3 \%)$, non-English speaking $(1.0 \%)$ or 
'Other' (27.1\%). Free text responses to the 'Other' option were varied, but common answers were that their child was planning to do it later, needed assistance, could not read, or was unwell or too distressed.

Six hundred and forty two children completed their episode of care at the pain management service during the period of data collection. Of these, 206 children and 199 parents completed both a referral questionnaire and another at the end of their episode. Approximately two thirds of patients who had completed an episode of care, and their parents, had therefore not completed episode end questionnaires (Figure 2). Reasons include that they had not yet been sent the episode end questionnaires, had not yet returned them, or they were unwilling or unable to do so. Participating services have evolving procedures for determining when an episode of active care has been completed, for capturing data at the last face-to-face appointment or despatching the questionnaires post hoc, and for sending reminders to complete and return them. Questionnaire return rates are included in individual pain unit reports, enabling units to track how return rates change with process improvements, over successive years.

Figure 2 here

\section{Description of patients at referral}

The demographic characteristics and comorbidity profile of children at referral are shown in Table 3. Patients were generally referred to the children's pain management service by a specialist practitioner (70.9\%).

Table 3 here

At referral, 56.5\% of parents reported that their child had experienced pain for more than 12 months. Most also described their child's pain as 'always present' (70.3\%). Parents attributed the onset of their child's pain to injury (21.6\%), illness (15.9\%), after surgery (7.4\%), 'no known cause' $(40.0 \%)$ or 'other' (15.1\%). The main pain was most commonly experienced in the lower limbs 
(28.4\%), back (17.0\%), abdomen (16.4\%), and head (15.4\%). Approximately 1 in 4 patients (26\%) had pain in one region only, with the majority identifying multiple painful regions.

One third of the children were enrolled in primary school (Kindergarten to Year 6) and two thirds in secondary school (Years $7-12$ ). Children missed a significant number of school days in the previous school term ${ }^{1}$ as a result of their pain, with the number of days missed increasing with age. Young children missed an average of 10.2 days (20.4\%), children 13.0 days (26.0\%) and adolescents 16.9 days (33.8\%) of the school term. A small proportion (13.6\%) of adolescents were in paid employment, with pain affecting the number of hours that most $(69.2 \%)$ could work.

Many children were using medications often or daily at referral: $56.0 \%$ were using paracetamol often or daily, $44.8 \%$ anti-inflammatory medication, $32.7 \%$ anti-neuropathic medicines, $24.3 \%$ complementary or alternative medicines, $15.7 \%$ opioid-containing medication. They also had relatively high healthcare utilisation for age. In the preceding 3 months, on account of their pain, children had an average of 3.6 general practitioner visits, 3.0 medical specialist visits, 3.7 allied health appointments, 1.2 hospital emergency department presentations and 2.2 diagnostic tests.

Table 4 shows child and parent scores by child age group at referral for each of the assessment tools.

Table 4 here

Both self-report and parent-proxy pain intensity ratings indicate that on average the children's usual pain was in the moderate category, while pain at its worst was severe. The self-reported quality of life of the children in this cohort appears worse than that of healthy children, those with other chronic health conditions, ${ }^{66,68,69}$ and children with chronic pain reported in other studies using this measure. ${ }^{36,71}$ Both child and parent report showed a trend of lower health-related quality of life with increasing age group. Compared to their parent's ratings, children tended to report better quality of

\footnotetext{
${ }^{1}$ The school year in Australia and New Zealand is divided into four 'terms', each lasting approximately 10 weeks (50 school days).
} 
life overall and for most of the PedsQL subscales, consistent with cross-informant variance observed in other studies using this measure..$^{36,68,70,71}$

Scores on the FDI were most likely to fall in the moderate category, as is typically reported for children referred for specialist pain management. ${ }^{49}$ However, the average FDI scores of 25.7 for 8-12 year olds and 26.8 for $13-18$ year olds were at the high end of the moderate disability range (13 to 29), and somewhat higher than those described elsewhere. ${ }^{48,49,70}$ For example, Kashikar-Zuck et al's study of a large paediatric pain population reported mean scores of 19.1 and 21.7 for 8-12 and 13-19 year olds, respectively. Pain-specific anxiety, measured by the Bath Adolescent Pain Questionnaire, was similar to that reported in other of studies of paediatric chronic pain patients. 5,35

\section{Patient outcomes}

During this period, 642 patients completed their treatment at the pain management service, representing $44.8 \%$ of the total patients registered. Approximately one third of these patients $(n=206,32.1 \%)$ and their carers $(n=199,31.0 \%)$ completed questionnaires both at referral and at the end of their episode. T- and z-tests showed that these children did not differ significantly from the larger population of children registered in PaedePPOC in terms of gender, age, country of birth, or scores at referral on all assessment tools (all $p$ values $>0.05$ ).

The referral and episode end scores for these children are shown in Table 5.

Table 5 here

In this small sub-sample with follow-up data, and using the clinically significant criteria described previously, $49.3 \%$ of children and $48.7 \%$ of parents reported that the child's average pain improved by at least $30 \%$ from referral to episode end. Change in the child's health-related quality of life reached clinical significance according to $68.9 \%$ of child-reports and $63.4 \%$ of parent-reports. Over half (58.9\%) of the children experienced a clinically significant improvement in their functional ability. There was a statistically significant decrease in scores measuring pain-specific anxiety $(t=8.60$, 
$p<0.0001$ ), and $48.4 \%$ of adolescents reported at least a $30 \%$ improvement from referral to episode end.

These improvements were accompanied by a reduction in the number of school days missed due to pain. At referral children missed a median of 10 days in the previous school term (interquartile range 4-21 days) and at episode end these figures were 5 days (0-15 days). Reductions in medications used often or daily for pain at referral and episode end are shown in Table 6.

Table 6 here 


\section{DISCUSSION [1525/1500 words]}

This foundation paper describes the processes whereby the Australian and New Zealand electronic Persistent Pain Outcomes Collaboration for children's specialist pain services (PaedePPOC) was established. Years of advocacy by professional and consumer groups, together with seed funding from the NSW Ministry of Health, catalysed the process. Thereafter, consensus on the PaedePPOC dataset, and roll-out of protocols, software and training were achieved within 18 months. Nine of the ten specialist paediatric pain services in Australia and New Zealand are now participating in PaedePPOC.

A number of factors facilitated the establishment and implementation of PaedePPOC. The most important of these was the parallel establishment of the adult pain outcomes collaboration. ${ }^{65}$ The synergy between the two meant that critical infrastructure and processes were already in place and could be modified rather than developed anew. For example, the epiCentre database, adult ePPOC service-level reports, training and support processes were amended to address the needs of the paediatric services. This also significantly reduced the cost of implementing PaedePPOC.

A second factor facilitating implementation was the relatively small number of children's pain services in Australasia, enabling all services to participate in meetings to discuss the development of PaedePPOC. Arriving at consensus on the minimum dataset was therefore able to occur relatively quickly. The small number of services also meant that existing ePPOC staff could accommodate the training, support and reporting requirements of PaedePPOC.

Other factors perhaps less unique to the Australasian environment were: 1) the importance placed on the dataset being useful for clinical purposes, with reporting, benchmarking and research as secondary uses of the data; 2) the development of purpose-built software for the collection and clinical use of the information; and 3) ongoing support and contact with the pain management units. Each of these helped to ensure focus and engagement of pain service staff with PaedePPOC, and to embed use of the questionnaires and software into routine clinical practice. 
There have been a number of benefits arising from the implementation of PaedePPOC. At the national level, the data have permitted a detailed description of the children referred for specialist pain management. Although limited, outcome data also indicate that a large proportion of the children who completed their treatment made important positive change in the intensity of pain experienced, their functional ability and health-related quality of life. The comparison of our population and outcomes with those described in other countries and settings will allow and inform discussion of issues including referral patterns, treatments provided and availability of services in this country compared to others.

At the service level, the individual pain unit reports have allowed each service to better understand the children they serve and to compare them to those seen elsewhere in Australia and in other countries. This latter comparison may be particularly useful in determining the extent to which scientific evidence derived in other countries and cultures (for example ${ }^{38}$ ) might be generalizable to the Australasian or individual service contexts.

The process of embedding PaedePPOC into service 'standard business' has yielded other, unforeseen benefits, beyond the information gained. The need to standardise terms to describe episodes, care pathways and service events has led to rich discussion and acknowledgement of the variation in practice across units. Having to label points of care as being on one pathway or another, or identifying the end of an episode of active care, required service teams to reflect and make explicit any plan changes. When an episode of active care is ended explicitly, this raises the probability that there will follow a clear hand-over of care to the referring doctor, complete with follow-on care advice and contingency plans for children and families.

The use of individual patient reports and graphics in conversations with young people and/or parents has also acknowledged, in a real way, consumer-ownership of their healthcare information, and improved engagement in treatment. This is consistent with the findings of a recent study highlighting the benefits of discussing patient-rated outcomes with the patient. ${ }^{32}$ Within the 
children's services, completion of subsequent questionnaires may also be more likely if the child and parent see that their responses inform treatment and are used to monitor progress. Another hypothesis is that PaedePPOC participation increases child and family sense of their own altruistic contribution to a greater good, and may contribute to post-traumatic growth.

While PaedePPOC has been embraced by children's specialist pain services in Australasia, its implementation has not been without challenges. A number of these were mentioned previously, including the difficulties deploying epiCentre, additional burden of data entry for pain service staff, and reconciling an existing service's processes and procedures with the PaedePPOC protocol.

Another ongoing challenge and important limitation in the data currently collected is that it may not reflect all patients referred for pain management. While the expectation of PaedePPOC is that each pain management service will submit data for all patients, the current data indicate that just over $75 \%$ of children and parents completed a referral questionnaire. There may be valid reasons for a proportion of these non-completions, for example, questionnaires not received at the time of data extraction, or referred patients failing to attend. In addition, because baseline and outcome information is self- or parent-reported, it is only available if the individuals are able to complete the questionnaires. PaedePPOC is looking to address these issues by promoting the use of questionnaires available in other languages, and investigating processes within each hospital to help ensure patient information is collected for all patients. It is possible, however, that people with low literacy or intellectual impairment will be underrepresented in the PaedePPOC dataset.

Another perennial challenge for outcome registries is to capture data from patients at and after discharge from active care. ${ }^{7,65}$ This is clearly apparent in the PaedePPOC data reported here, with a small number of episode outcomes available relative to the number of children referred. The reasons for this may include losing contact with patients and limited availability of resources to send and monitor questionnaires. However we believe that one of the primary reasons, which became apparent in the early stages of both PaedePPOC and ePPOC, was that episodes of specialist pain care 
were often lengthy or nebulous. Many services did not discharge patients, while others had no clear process for identifying when treatment was complete. This meant that a measure of treatment outcome may never be obtained for a large proportion of patients. In order to address this and maximise the number of episode outcomes that could be obtained, a definitional change to the 'end of episode' was made. Whereas an episode end was previously defined as the point of discharge from a service, the definition now relates to when 'active treatment' is complete. This may be at the end of a defined treatment (e.g. a group pain program or series of individual appointments) or when the treating clinicians reduce the frequency of appointments from therapy to maintenance levels. It is expected that this will lead to an increased proportion of episode closures and outcomes in the future. Outcome data is critical to the aims of PaedePPOC, and the ongoing viability of the collaboration will depend on how successful we are in our ability to gather this information in future. One further challenge is assuring the financial sustainability of the collaboration. ePPOC and PaedePPOC are jointly funded by the primary stakeholders - individual pain management services, state governments, and insurance and compensation organisations. These organisations fund the maintenance of the ePPOC/PaedePPOC infrastructure and staffing necessary to carry out the functions of the collaborations. In addition, participating services must manage the sustainability of their own participation including managing communications with patients, data entry and extraction, attendance at PaedePPOC meetings, and managing review of service reports as part of internal quality improvement activities. For the sector and consumers to benefit from PaedePPOC into the future, its financial and service-level sustainability must remain a focus area.

The years 2014 to 2018 have marked the establishment phase of PaedePPOC, with the emphasis on dataset development and integration into clinical processes. The next three years will focus on increasing collection of outcome data and establishing benchmarks for the sector. Benchmarks have been created for the adult pain services and will be used in PaedePPOC to identify reasons for variation between services and the practices and processes that result in the best outcomes for patients. As baseline and outcome data builds there will also be an increased focus on use of the 
data for research. For example, future research will examine concordance between self- and proxyreports, identification of children whose outcomes are positive compared to those that are not including the factors related to or predictive of these, and use of the patient linkage key in PaedePPOC and EPPOC to identify those children who transition to or re-appear in adult services This paper describes the evolution of PaedePPOC in services across Australia and New Zealand. We hope that service clinicians and planners may see in this description, elements that could inform the development of persistent pain outcome registries in other jurisdictions. The drivers, collaborative processes, and dataset may be relevant or adapted to other, similar initiatives. Ultimately, international collaboration will be facilitated by gradually aligning outcome measurement suites across borders. 


\section{Acknowledgements}

The establishment of ePPOC was funded by the New South Wales Ministry of Health. The authors acknowledge members of the ePPOC Paediatric Reference Group and colleagues who contributed in person or via correspondence to the minimum dataset consensus. The authors also acknowledge staff of participating services and consumer representatives who provided valuable feedback during development and implementation. We would like to thank staff at the participating pain management services for collecting and collating the data used in this paper. 


\section{REFERENCES}

1. Access Economics Pty Limited for MBF Foundation in Collaboration with University of Sydney Pain Management Research Institute. The high price of pain: the economic impact of persistent pain in Australia. 2007. [online] pp.1-88. Available at:

http://www.painaustralia.org.au/images/pain australia/MBF\%20Economic\%20Impact.pdf

2. Antonaci F, Voiticovschi-losob C, Di Stefano AL, Galli F, Ozge A, Balottin U. The evolution of headache from childhood to adulthood: a review of the literature. The Journal of Headache and Pain 2014;15:15-26.

3. Australian Health Services Research Institute. About AHSRI. https://ahsri.uow.edu.au (accessed Oct 2017).

4. Australian Pain Society. Summary of the Australian Pain Society Achievements to date and their alignment with the Goals of the National Pain Strategy.

https://www.apsoc.org.au/Achievements and https://www.apsoc.org.au/pain-in-childhood (accessed Oct 2017).

5. Benore E, D'Auria A, Banez GA, Worley S, Tang A. The influence of anxiety reduction on clinical response to pediatric chronic pain rehabilitation. Clin J Pain 2015;31:375-383.

6. Berde CB, Walco GA, Krane EJ, Anand KJ, Aranda JV, Craig KD, Dampier CD, Finkel JC, Grabois M, Johnston C, Lantos J, Lebel A, Maxwell LG, McGrath P, Oberlander TF, Schanberg LE, Stevens B, Taddio A, von Baeyer CL, Yaster M, Zempsky WT. Pediatric analgesic clinical trial designs, measures, and extrapolation: report of an FDA scientific workshop. Pediatrics 2012;129(2):354-64 .

7. Bhandari RP, Feinstein AB, Huestis SE, Krane EJ, Dunn AL, Cohen LL, Kao MC, Darnall BD, Mackey SC. Pediatric-Collaborative Health Outcomes Information Registry (Peds-CHOIR): A learning health system to guide pediatric pain research and treatment. Pain 2016;157(9):2033-2044. 
8. Birnie KA, Hundert AS, Lalloo C, Nguyen C, Stinson JN. Recommendations for selection of self-report pain intensity measures in children and adolescents: a systematic review and quality assessment of measurement properties. Pain 2019;160:5-18.

9. Blyth FM, March LM, Brnabic AJM, Jorm LR, Williamson M, Cousins MJ. Chronic pain in Australia: a prevalence study. Pain 2001;89:127-134.

10. Blyth FM, Van Der Windt DA, Croft PR. Chronic disabling pain: a significant public health problem. Am J Prev Med 2015;49:98-101.

11. Breivik H, Eisenberg E, O'Brien T. The individual and societal burden of chronic pain in Europe: the case for strategic prioritisation and action to improve knowledge and availability of appropriate care. BMC Public Health. 2013;13:1229.

12. Campbell F, Stinson J, Ouellette C, Ostapets V, Salibury G. The association between pediatric chronic pain clinic attendance and health care utilization: A retrospective analysis. Can J Pain 2018;2:30-36.

13. Choinière $M$, Ware $M A$, Pagé $M G$, Lacasse $A$, Lanctôt $H$, Beaudet $N$, Boulanger $A$, Bourgault P, Cloutier C, Coupal L, De Koninck Y, Dion D, Dolbec P, Germain L, Martin V, Sarret P, Shir Y, Taillefer M-C, Tousignant B, Trépanier A, Truchon R. Development and implementation of a registry of patients attending multidisciplinary pain treatment clinics: the Quebec Pain Registry. Pain Research and Management 2017; Article ID 8123812, 16 pages http://dx.doi.org/10.1155/2017/8123812

14. Cleeland CS, Ryan KM. Pain assessment: global use of the Brief Pain Inventory. Annals of the Academy of Medicine Singapore. 1994;23:129-38.

15. Cooper TE, Fisher E, Anderson B, Wilkinson NMR, Williams DG, Eccleston C. Paracetamol (acetaminophen) for chronic non-cancer pain in children and adolescents. Cochrane Database of Systematic Reviews 2017, Issue 8. Art. No.: CD012539. DOI: 10.1002/14651858.CD012539.pub2. 
16. Cooper TE, Fisher E, Gray AL, Krane E, Sethna N, van Tilburg MAL, Zernikow B, Wiffen PJ. Opioids for chronic non-cancer pain in children and adolescents. Cochrane Database of Systematic Reviews 2017, Issue 7. Art. No.: CD012538. DOI:

10.1002/14651858.CD012538.pub2.

17. Cooper TE, Heathcote LC, Clinch J, Gold J, Howard R, Lord SM, Schechter N, Wood C, Wiffen PJ. Antidepressants for chronic non-cancer pain in children and adolescents. Cochrane Database of Systematic Reviews 2017, Issue 8. Art. No.: CD012535. DOI: 10.1002/14651858.CD012535.pub2.

18. Cooper TE, Wiffen PJ, Heathcote LC, Clinch J, Howard R, Krane E, Lord SM, Sethna N, Schechter N, Wood C. Antiepileptic drugs for chronic non-cancer pain in children and adolescents. Cochrane Database of Systematic Reviews 2017, Issue 8. Art. No.: CD012536. DOI: 10.1002/14651858.CD012536.pub2.

19. Dick BD, Pillai Riddell R. Cognitive and school functioning in children and adolescents with chronic pain: A critical review. Pain Res Manage 2010;15:238-244.

20. Dominick C, Blyth F, Nicholas M. Patterns of chronic pain in the New Zealand population. N Z Med J 2011;124:63-76. https://www.nzma.org.nz/journal/read-the-journal/all-issues/20102019/2011/vol-124-no-1337/article-dominick

21. Donaldson LJ. Pain: breaking through the barrier. 150 years of the Annual Report of the Chief Medical Officer: on the state of the public health. London, 2008.

http://webarchive.nationalarchives.gov.uk/20130105021744/http://www.dh.gov.uk/en/Pub licationsandstatistics/Publications/AnnualReports/DH 096206

22. Dworkin RH, Turk DC, Farrar JT, Haythornthwaite JA, Jensen MP, Katz NP, Kerns RD, Stucki G, Allen RR, Bellamy N, Carr DB, Chandler J, Cowan P, Dionne R, Galer BS, Hertz S, Jadad AR, Kramer LD, Manning DC, Martin S, McCormick CG, McDermott MP, McGrath P, Quessy S, Rappaport BA, Robbins W, Robinson JP, Rothman M, Royal MA, Simon L, Stauffer JW, Stein 
W, Tollett J, Wernicke J, Witter J; IMMPACT. Core outcome measures for chronic pain clinical trials: IMMPACT recommendations. Pain 2005;113:9-19.

23. Eccleston C, Clinch J. Adolescent chronic pain and disability: A review of the current evidence in assessment and treatment. Paediatr Child Health 2007;12:117-20.

24. Eccleston C, Cooper TE, Fisher E, Anderson B, Wilkinson NMR. Non-steroidal antiinflammatory drugs (NSAIDs) for chronic non-cancer pain in children and adolescents. Cochrane Database of Systematic Reviews 2017, Issue 8. Art. No.: CD012537. DOI: 10.1002/14651858.CD012537.pub2.

25. Eccleston C, Fisher E, Law E, Bartlett J, Palermo TM. Psychological interventions for parents of children and adolescents with chronic illness. Cochrane Database of Systematic Reviews 2015 , Issue 4 . Art. No.: CD009660. DOI: 10.1002/14651858.CD009660.pub3.

26. Eccleston C, Jordan A, McCracken LM, Sleed M, Connell H, Clinch J. The Bath Adolescent Pain Questionnaire (BAPQ): Development and preliminary psychometric evaluation of an instrument to assess the impact of chronic pain on adolescents. Pain 2005;118:263-270.

27. Eccleston C, Palermo TM, Williams AC de C, Lewandowski Holley A, Morley S, Fisher E, Law E. Psychological therapies for the management of chronic and recurrent pain in children and adolescents. Cochrane Database of Systematic Reviews 2014, Issue 5. Art. No.: CD003968. DOI: 10.1002/14651858.CD003968.pub4.

28. Electronic Persistent Pain Outcomes Collaboration. ePPOC Governance. https://ahsri.uow.edu.au/eppoc/governance/index.html (accessed Mar 2018)

29. Electronic Persistent Pain Outcomes Collaboration. ePPOC Reports. https://ahsri.uow.edu.au/eppoc/reports/index.html (accessed Mar 2018)

30. Fayaz A, Croft P, Langford RM, et al. Prevalence of chronic pain in the UK: a systematic review and meta-analysis of population studies. BMJ Open 2016;6:e010364.

31. Fisher E, Law E, Palermo TM, Eccleston C. Psychological therapies (remotely delivered) for the management of chronic and recurrent pain in children and adolescents. Cochrane 
Database of Systematic Reviews 2015, Issue 3. Art. No.: CD011118. DOI:

10.1002/14651858.CD011118.pub2.

32. Freel J, Bellon J, Hanmer J. Better physician ratings from discussing PROs with patients. NEJM Catalyst 2018; June 20. https://catalyst.nejm.org/ratings-patients-discussing-pros/ (accessed July 2018)

33. Gagnon C, Amstutz D, Revivo G. Psychometric evaluation of the Bath Adolescent Pain Questionnaire (BAPQ) in a US-based clinical population. J Pain 2011;12(4,Supplement):P17.

34. Gaskin DJ, Richard P. The economic costs of pain in the United States. J Pain 2012;13:715-24.

35. Gauntlett-Gilbert J, Connell H, Clinch J, McCracken LM. Acceptance and values-based treatment of adolescents with chronic pain: outcomes and their relationship to acceptance. Journal of Pediatric Psychology 2013;38:72-81.

36. Gold JI, Mahrer NE, Yee J, Palermo TM. Pain, fatigue and health-related quality of life in children and adolescents with chronic pain Clin J Pain 2009;25:407-412.

37. Groenewald CB, Wright DR, Palermo TM. Health care expenditures associated with pediatric pain-related conditions in the United States. Pain 2015;156:951-957.

38. Hechler T, Kanstrup M, Lewandowski Holley A, Simons LE, Wicksell R, Hirschfeld G, Zernikow B. Systematic review on intensive interdisciplinary pain treatment of children with chronic pain. Pediatr 2015;136(1):115-127.

39. Henderson JV, Harrison CM, Britt HC, Bayram CF, Miller GC. Prevalence, causes, severity, impact, and management of chronic pain in Australian general practice patients. Pain Medicine 2013;14:1346-1361.

40. Hestbaek L, Leboeuf-Yde C, Kyvik KO, Manniche C: The course of low back pain from adolescence to adulthood: eight-year follow-up of 9600 twins. Spine 2006;31:468-472.

41. Hicks CL, von Baeyer CL, Spafford P, van Korlaar I, Goodenough B. The Faces Pain Scale Revised: Toward a common metric in pediatric pain measurement. Pain, 2001;93:173-183. (c) 2001, International Association for the Study of Pain. www.iasp-pain.org/RPSR 
42. Hogan ME, Taddio A, Katz J, Shah V, Krahn M. Incremental health care costs for chronic pain in Ontario, Canada: a population-based matched cohort study of adolescents and adults using administrative data. Pain. 2016;157:1626-1633.

43. Hogg MN, Gibson S, Helou A, DeGabriele J, Farrell MJ. Waiting in pain: a systematic investigation into the provision of persistent pain services in Australia. Med J Aust 2012;196:386-390.

44. Hoque DME, Kumari V, Hoque M, Ruseckaite R, Romero L, Evans SM. Impact of clinical registries on quality of patient care and clinical outcomes: A systematic review. PLOS ONE 2017;12:e0183667. https://doi.org/10.1371/journal.pone.0183667

45. Institute of Medicine. Relieving Pain in America: A Blueprint for Transforming Prevention, Care, Education, and Research. Washington, DC: The National Academies Press, 2011.

46. Jones K, Nordstokke D, Wilcox G, Schroeder M, Noel M. The 'work of childhood': understanding school functioning in youth with chronic pain. Pain Management 2018;8:139153. doi: 10.2217/pmt-2017-0048. Epub 2018 Feb 22.

47. Jordan A, Eccleston C, McCracken LM, Connell H, Clinch J. The Bath Adolescent Pain Parental Impact Questionnaire (BAP-PIQ): development and preliminary psychometric evaluation of an instrument to assess the impact of parenting an adolescent with chronic pain. Pain, 2008;137:478-487. Bath Centre for Pain Research www.bath.ac.uk/pain

48. Kaczynski KJ, Simons LE, Lewis Claar R. Anxiety, coping, and disability: a test of mediation in a pediatric chronic pain sample. Journal of Pediatric Psychology 2011;36:932-941.

49. Kashikar-Zuck S, Flowers SR, Lewis Claar R, Guite JW, Logan DE, Lynch-Jordan AM, Palermo TM, Wilson AC. Clinical utility and validity of the Functional Disability Inventory (FDI) among a multicenter sample of youth with chronic pain. Pain 2011;152:1600-1607.

50. King S, Chambers $\mathrm{CT}$, Huguet $\mathrm{A}$, et al. The epidemiology of chronic pain in children and adolescents revisited: a systematic review. Pain 2011;152:2729-2738. 
51. Larsson S, Lawyer P, Garellick G, Lindahl B, Lundström M. Use of 13 disease registries in 5 countries demonstrates the potential to use outcome data to improve health care's value. Health Affairs 2012;1:220-227.

52. Mackey S, Kao MC, Cook K, Olson G, Pacht T, Darnall B, Weber S. Collaborative Health Outcomes Information Registry (CHOIR): Open source cloud based platform to generate and support learning healthcare systems. Postgraduate Medicine, Pain Management 2014;66.

53. McGrath PJ, Walco GA, Turk DC, Dworkin RH, Brown MT, Davidson K, Eccleston C, Finley GA, Goldschneider K, Haverkos L, Hertz SH, Ljungman G, Palermo T, Rappaport BA, Rhodes T, Schechter N, Scott J, Sethna N, Svensson OK, Stinson J, von Baeyer CL, Walker L, Weisman S, White RE, Zajicek A, Zeltzer L. Core outcome domains and measures for pediatric acute and chronic/recurrent pain clinical trials: PedIMMPACT recommendations. J Pain 2008;9:771783.

54. Miró J, Castarlenas E, Huguet A. Evidence for the use of a numerical rating scale to assess the intensity of pediatric pain. Eur J Pain 2009;13:1089-1095.

55. Nahin RL. Estimates of Pain Prevalence and Severity in Adults: United States, 2012. J Pain 2015;16:769-780.

56. National Health Data Dictionary. Cat. No. HWI, 119th edition. Canberra, Australia: Australian Institute of Health and Welfare, 2012.

57. National Pain Audit Final Report 2010-2012. Healthcare Quality Improvement Partnership. Britain, 2012. https://www.hqip.org.uk/resources/former-hqip-commissionedprogrammes/national-pain-audit/ (accessed Jan 2018)

58. National Pain Summit Initiative. National Pain Strategy. Canberra, ACT: Pain Australia, 2011, pp 46-47. http://www.painaustralia.org.au/static/uploads/files/national-pain-strategy-2011wfvjawttsanq.pdf (accessed Jan 2018)

59. NSW Ministry of Health - Health and Social Policy. Report of the NSW Pain Management Taskforce. Sydney, NSW: NSW Government, 2012, p51. ISBN 978-1-74187-784-7 
http://www.health.nsw.gov.au/PainManagement/Publications/NSW-pain-management-

report.pdf (accessed Oct 2017)

60. O'Sullivan PB, Beales DJ, Smith AJ, Straker LM. Low back pain in 17 year olds has substantial impact and represents an important public health disorder: a cross-sectional study. BMC Public Health 2012;12:100.

61. Sato AF, Hainsworth KR, Khan KA, Ladwig RJ, Weisman SJ, Davies WH. School absenteeism in pediatric chronic pain: Identifying lessons learned from the general school absenteeism literature. Child Health Care 2007;36:355-72.

62. Sil S, Arnold LM, Lynch-Jordan A, Ting TV, Peugh J, Cunningham N, Powers SW, Lovell DJ, Hashkes PJ, Passo M, Schikler KN, Kashikar-Zuck S. Identifying Treatment Responders and Predictors of Improvement after Cognitive-Behavioral Therapy for Juvenile Fibromyalgia. Pain 2014;155:1206-1212.

63. Sleed M, Eccleston C, Beecham J, Knapp M, Jordan A: The economic impact of chronic pain in adolescence: methodological considerations and a preliminary costs-of-illness study. Pain 2005;119:183-190.

64. Swain MS, Henschke N, Kamper SJ, Gobina I, Ottova-Jordan V, Maher CG. An international survey of pain in adolescents. BMC Public Health. 2014;4:447.

65. Tardif H, Arnold C, Hayes C, Eagar K. Establishment of the Australasian Electronic Persistent Pain Outcomes Collaboration. Pain Medicine 2016;0:1-12. doi: 10.1093/pm/pnw201

66. Varni JW, Burwinkle TM. The PedsQL $\mathrm{L}^{\mathrm{TM}}$ as a patient-reported outcome in children and adolescents with Attention-Deficit/Hyperactivity Disorder: a population-based study. Health and Quality of Life Outcomes 2006;4:26-35.

67. Varni JW, Burwinkle TM, Seid M. The PedsQL as a Pediatric Patient-Reported Outcome: Reliability and Validity of the PedsQL Measurement Model in 25,000 Children. Expert Review of Pharmacoeconomics Outcomes Research 2005;5:705-718. ㄷ 1998 JW Varni PhD. All rights reserved. 
68. Varni JW, Burwinkle TM, Seid M, Skarr D. The PedsQL ${ }^{\mathrm{TM}} 4.0$ as a Pediatric Population Health Measure: Feasibility, Reliability, and Validity. Ambulatory Pediatrics 2003;3:329-341.

69. Varni JW, Limbers CA, Burwinkle TM. Impaired health-related quality of life in children and adolescents with chronic conditions: a comparative analysis of 10 disease clusters and 33 disease categories/severities utilizing the PedsQL ${ }^{\text {TM }}$ 4.0 Generic Core Scales. Health and Quality of Life Outcomes 2007;5:43-54.

70. Vetter TR, Bridgewater CL, Ascherman LI, Madan-Swain A, McGwin GL. Patient versus parental perceptions about pain and disability in children and adolescents with a variety of chronic pain conditions. Pain Res Manag 2014;19(1):7-14.

71. Vetter TR, Bridgewater CL, McGwin G. An observational study of patient versus parental perceptions of health-related quality of life in children and adolescents with a chronic pain condition: who should the clinician believe? Health and Quality of Life Outcomes 2012;10:85-96.

72. von Baeyer CL, Lin V, Seidman LC, Tsao JC, Zeltzer LK. Pain charts (body maps or manikins) in assessment of the location of pediatric pain. Pain Manag 2011;1(1):61-68. DOI: $10.2217 /$ pmt.10.2

73. von Baeyer CL, Spagrud LJ, McCormick JC, Choo E, Neville K, Connelly MA. Three new datasets supporting use of the Numerical Rating Scale (NRS-11) for children's self-reports of pain intensity. Pain 2009;143:223-227.

74. Walker LS, Dengler-Crish CM, Rippel S, Bruehl S. Functional abdominal pain in childhood and adolescence increases risk for chronic pain in adulthood. Pain 2010;150:568-572. doi:10.1016/j.pain.2010.06.018

75. Walker LS, Greene JW. The Functional Disability Inventory (FDI): Measuring a neglected dimension of child health status. Journal of Paediatric Psychology 1991;16:39-58.

76. Wilcox N, McNeil JJ. Clinical quality registries have the potential to drive improvements in the appropriateness of care. Med J Aust 2016;205:S21-S26. 
Figure 1. Graphic representation of a 13 year old child's self-reported progress.

The top left panel shows pain severity 'in the last week' rated at referral and review dates using the modified- $\mathrm{BPI}$, where $0=$ 'no pain' and $10=$ 'pain as bad as you can imagine'. The top right panel shows quality of life 'in the last month' using PedsQL subscale and total scores, where $0=$ all activities rated as 'almost always' a problem, and $100=$ all activities rated as 'never' a problem. The bottom left panel shows functional disability 'in the last two weeks' using FDI scores at referral and review overlying FDI disability severity bands. An FDI score of $0=$ all 15 physical activities were rated as being 'no trouble', and $60=$ all activities were rated as 'impossible'. The bottom right panel shows pain-related worries 'in the last two weeks' rated on the BAPQ Section 5 , where $0=$ all seven worries/thoughts scored as 'never' experienced, and $28=$ all seven worries/thoughts scored as 'always' experienced. These graphics can be used to discuss progress with the young person and their family in clinic, and may be included in clinic letters to update the referring practitioner. 
Figure 2. Data collection flow diagram. 
Table 1. Data collection domains and their sources

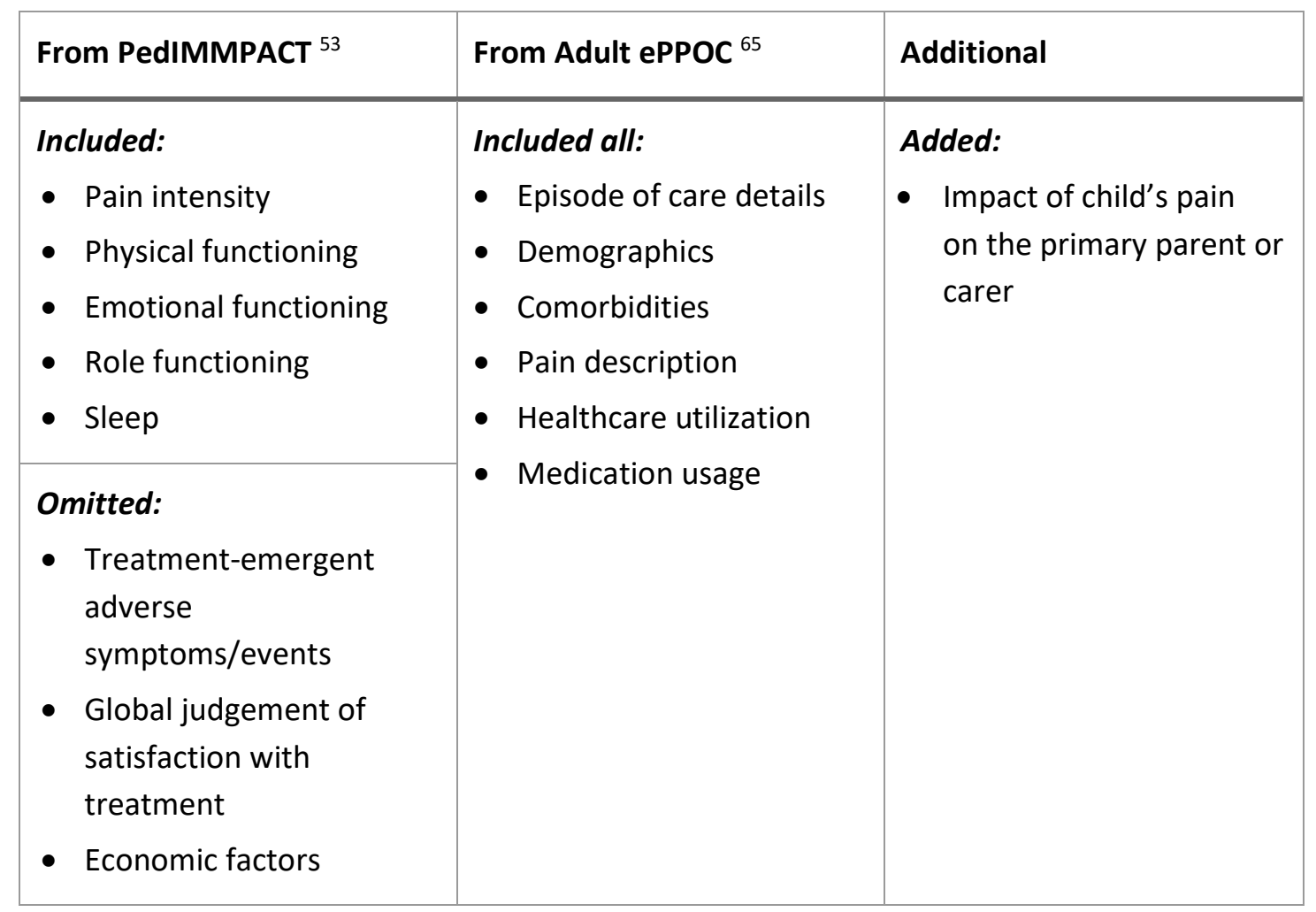


Table 2. Measurement tools used in Paed-ePPOC

\begin{tabular}{|c|c|c|c|}
\hline Domain & Variable & Measurement Tool(s) & $\begin{array}{l}\text { Age group } \\
\text { (Source) }\end{array}$ \\
\hline \multirow[t]{4}{*}{ Pain } & Context & $\begin{array}{l}\text { Categorical options for how pain began, } \\
\text { duration since onset, and association with } \\
\text { cancer or compensation }\end{array}$ & All (P) \\
\hline & Location & $\begin{array}{l}\text { CARRA Body-chart for worst pain site and all } \\
\text { pain sites }\end{array}$ & All $(C+P)$ \\
\hline & Frequency & $\begin{array}{l}\text { Five categorical options - always present with } \\
\text { constant intensity, always present but varying } \\
\text { intensity, often, occasionally or rarely present }\end{array}$ & $\begin{array}{l}5-7 \text { years }(P) \\
8-12 \text { years }(C+P) \\
13-18 \text { years }(C+P)\end{array}$ \\
\hline & Intensity & $\begin{array}{l}\text { Pain now, and best, worst and usual pain during } \\
\text { the last week using either the Faces of Pain } \\
\text { Scale-R for children 5-7 years or Modified Brief } \\
\text { Pain Inventory for all older children and parent } \\
\text { report }\end{array}$ & All $(C+P)$ \\
\hline \multirow[t]{2}{*}{$\begin{array}{l}\text { Physical } \\
\text { functioning }\end{array}$} & $\begin{array}{l}\text { Severity of } \\
\text { limitation }\end{array}$ & Functional Disability Inventory & $\begin{array}{l}8-12 \text { years }(C) \\
13-18 \text { years }(C)\end{array}$ \\
\hline & $\begin{array}{l}\text { Frequency of } \\
\text { limitation }\end{array}$ & Pediatric Quality of Life physical function & All $(C+P)$ \\
\hline \multirow[t]{2}{*}{$\begin{array}{l}\text { Emotional } \\
\text { functioning }\end{array}$} & $\begin{array}{l}\text { Pain-related } \\
\text { worries }\end{array}$ & $\begin{array}{l}\text { Bath Adolescent Pain Questionnaire (Section } 5 \\
\text { - pain-related worry) }\end{array}$ & $13-18$ years $(C)$ \\
\hline & $\begin{array}{l}\text { Frequency of other } \\
\text { negative emotions }\end{array}$ & Pediatric Quality of Life emotional function & All $(C+P)$ \\
\hline \multirow{3}{*}{$\begin{array}{l}\text { Role } \\
\text { functioning }\end{array}$} & School attendance & School days missed & All (P) \\
\hline & Work capacity & $\begin{array}{l}\text { If child was employed, reduction in work hours } \\
\text { due to pain }\end{array}$ & $13-18$ years $(P)$ \\
\hline & $\begin{array}{l}\text { School and peer } \\
\text { function }\end{array}$ & $\begin{array}{l}\text { Pediatric Quality of Life school and peer } \\
\text { function }\end{array}$ & All $(C+P)$ \\
\hline Sleep & Trouble sleeping & $\begin{array}{l}\text { Pediatric Quality of Life single question about } \\
\text { sleep }\end{array}$ & All $(C+P)$ \\
\hline $\begin{array}{l}\text { Medication } \\
\text { usage }\end{array}$ & $\begin{array}{l}\text { Frequency of use of } \\
\text { key drug groups }\end{array}$ & $\begin{array}{l}\text { Categorical options to indicate use daily, often, } \\
\text { sometimes, rarely, never }\end{array}$ & All (P) \\
\hline $\begin{array}{l}\text { Healthcare } \\
\text { Utilization }\end{array}$ & $\begin{array}{l}\text { Utilization of health } \\
\text { care services in the } \\
\text { last } 3 \text { months }\end{array}$ & $\begin{array}{l}\text { Questions regarding general practitioner, } \\
\text { specialist, allied health, alternative therapists, } \\
\text { emergency department visits, admissions to } \\
\text { hospital and diagnostic test usage in the last } \\
\text { three months }\end{array}$ & All (P) \\
\hline \multirow{2}{*}{$\begin{array}{l}\text { Parent impact } \\
\text { of child's pain }\end{array}$} & Work capacity & Reduced hours & All (P) \\
\hline & $\begin{array}{l}\text { Impact on parental } \\
\text { mood, cognitions, } \\
\text { relationships }\end{array}$ & $\begin{array}{l}\text { Bath Adolescent Pain Parent Impact } \\
\text { Questionnaire }\end{array}$ & All (P) \\
\hline
\end{tabular}

C - child/adolescent self-report; CARRA - Childhood Arthritis and Rheumatology Research Alliance; $\mathrm{P}$ - parent proxy-report 
Table 3. Demographic information and comorbidities of children at referral

\begin{tabular}{|c|c|}
\hline \multicolumn{2}{|l|}{ Gender, $N(\%)(n=1432)+$} \\
\hline - Male & $452(31.6)$ \\
\hline - Female & $980(68.4)$ \\
\hline \multicolumn{2}{|l|}{ Age in years, mean (SD) ( $n=1432)$} \\
\hline - Male & $11.7(3.2)$ \\
\hline - Female & $12.8(2.9)$ \\
\hline \multicolumn{2}{|l|}{ Age group, $N(\%)(n=1432)$} \\
\hline$-<5$ years & $23(1.6)$ \\
\hline$-5-7$ years & $73(5.1)$ \\
\hline$-8-12$ years & $523(36.5)$ \\
\hline$-13-18$ years & $813(56.8)$ \\
\hline \multicolumn{2}{|l|}{ Country of birth, $N(\%)(n=1141)$} \\
\hline - Australia & $1063(93.2)$ \\
\hline - Other & $78(6.8)$ \\
\hline Aboriginal and/or Torres Strait Islander, N (\%) ( $n=1020)$ & $52(5.1)$ \\
\hline \multicolumn{2}{|l|}{ Comorbidities and disabilities, N (\%) (n=1076) } \\
\hline - chronic disease & $224(20.8)$ \\
\hline - mental health condition & $256(23.8)$ \\
\hline - cancer & $20(1.9)$ \\
\hline - sight impairment & $71(6.6)$ \\
\hline - hearing impairment & $22(2.0)$ \\
\hline - intellectual disability & $23(2.1)$ \\
\hline - physical disability & $80(7.4)$ \\
\hline
\end{tabular}

$+n-$ Number of baseline questionnaires wherein the given demographic field was completed by respondents

SD - Standard deviation 
Table 4. Child and parent scores on assessment tools at referral, by age group

\begin{tabular}{|c|c|c|c|}
\hline \multicolumn{2}{|c|}{ Outcome measure } & \multicolumn{2}{|c|}{ Mean score (SD) } \\
\hline & & Child report & Parent report \\
\hline \multicolumn{2}{|c|}{ Young Children (5-7 years) } & $n=32$ & $n=70$ \\
\hline \multirow{2}{*}{ Pain Severity } & - Worst pain & $7.0(3.0)$ & $7.0(2.3)$ \\
\hline & - Average pain & $4.9(2.7)$ & $5.2(1.9)$ \\
\hline \multirow[t]{6}{*}{ PedsQL } & - Total score & $53.0(15.9)$ & $49.2(17.8)$ \\
\hline & - Emotional & $45.6(27.2)$ & $47.2(19.1)$ \\
\hline & - Social & $63.2(24.3)$ & $60.9(24.8)$ \\
\hline & - School & $58.1(21.3)$ & $54.8(21.1)$ \\
\hline & - Physical & $48.1(19.0)$ & $40.6(24.4)$ \\
\hline & - Sleep & $32.3(37.9)$ & $37.3(32.3)$ \\
\hline \multicolumn{2}{|c|}{ Children (8-12 years) } & $n=396$ & $n=403$ \\
\hline \multicolumn{2}{|c|}{ Pain Severity - Worst pain } & $7.6(2.1)$ & $7.6(2.2)$ \\
\hline & - Average pain & $5.7(2.4)$ & $5.5(2.1)$ \\
\hline \multirow[t]{6}{*}{ PedsQL } & - Total score & $50.8(17.5)$ & $47.6(17.1)$ \\
\hline & - Emotional & $49.8(22.4)$ & $46.7(20.8)$ \\
\hline & - Social & $65.9(21.5)$ & $61.8(21.6)$ \\
\hline & - School & $53.0(22.0)$ & $49.8(22.2)$ \\
\hline & - Physical & $40.8(23.9)$ & $37.9(23.9)$ \\
\hline & - Sleep & $32.7(32.1)$ & $34.7(31.7)$ \\
\hline \multicolumn{2}{|l|}{ FDI } & $25.7(12.0)$ & $\mathrm{n} / \mathrm{a}$ \\
\hline \multicolumn{2}{|c|}{ Adolescents (13-18 years) } & $n=648$ & $n=635$ \\
\hline \multirow[t]{2}{*}{ Pain Severity } & - Worst pain & $7.7(1.8)$ & $7.5(2.0)$ \\
\hline & - Average pain & $5.7(2.0)$ & $5.7(1.9)$ \\
\hline \multirow[t]{6}{*}{ PedsQL } & - Total score & $48.0(17.9)$ & $44.8(16.4)$ \\
\hline & - Emotional & $50.1(23.0)$ & $45.8(21.0)$ \\
\hline & - Social & $65.0(23.7)$ & $60.2(21.4)$ \\
\hline & - School & $44.0(22.7)$ & $41.5(21.5)$ \\
\hline & - Physical & $38.6(21.6)$ & $36.7(21.6)$ \\
\hline & - Sleep & $32.9(32.0)$ & $32.0(30.0)$ \\
\hline \multicolumn{2}{|l|}{ FDI } & $26.8(12.1)$ & $\mathrm{n} / \mathrm{a}$ \\
\hline \multicolumn{2}{|l|}{ BAP-Q5 } & $15.1(6.2)$ & $\mathrm{n} / \mathrm{a}$ \\
\hline
\end{tabular}

BAP-Q5 - Bath Adolescent Pain Questionnaire, Pain-related worry section; FDI - Functional Disability Inventory; $n$ - Number of baseline questionnaires wherein this field was validly completed by respondents; $n / a$ - Not applicable as parent-proxy questionnaires are not collected; PedsQL - Pediatric Quality of Life Inventory, Generic core scales; SD - Standard deviation 
Table 5. Scores at referral and episode end - mean (SD)

\begin{tabular}{|c|c|c|c|c|c|c|c|c|}
\hline & \multicolumn{4}{|c|}{ Child report } & \multicolumn{4}{|c|}{ Parent report } \\
\hline & $\mathrm{n}$ & $\begin{array}{l}\text { Referral } \\
\text { score }\end{array}$ & $\begin{array}{l}\text { Episode } \\
\text { end score }\end{array}$ & $\begin{array}{l}p \text {-valuet } \\
\text { for change }\end{array}$ & $\mathrm{n}$ & $\begin{array}{l}\text { Referral } \\
\text { score }\end{array}$ & $\begin{array}{l}\text { Episode } \\
\text { end score }\end{array}$ & $\begin{array}{l}p \text {-valuet } \\
\text { for change }\end{array}$ \\
\hline \multirow{3}{*}{$\begin{array}{l}\text { Pain Intensity } \\
\text { - Worst } \\
\text { - Average }\end{array}$} & & & & & & & & \\
\hline & 202 & $7.8(1.8)$ & $5.7(3.1)$ & $<0.0001$ & 188 & $7.7(2.0)$ & $5.3(3.1)$ & $<0.0001$ \\
\hline & 201 & $5.7(2.0)$ & $3.7(2.5)$ & $<0.0001$ & 187 & $5.6(1.8)$ & $3.7(2.3)$ & $<0.0001$ \\
\hline \multirow{7}{*}{$\begin{array}{l}\text { PedsQL } \\
\text { - Total } \\
\text { - Emotional } \\
\text { - Social } \\
\text { - School } \\
\text { - Physical } \\
\text { - Sleep }\end{array}$} & & & & & & & & \\
\hline & 196 & $47.6(17.0)$ & $64.1(21.6)$ & $<0.0001$ & 182 & $45.5(14.9)$ & $58.1(20.5)$ & $<0.0001$ \\
\hline & 193 & $49.3(22.1)$ & $66.4(23.8)$ & $<0.0001$ & 182 & $47.9(18.3)$ & $58.1(22.9)$ & $<0.0001$ \\
\hline & 195 & $63.3(23.3)$ & $72.7(22.8)$ & $<0.0001$ & 182 & $59.3(21.3)$ & 66.9 (23.6) & $<0.0001$ \\
\hline & 195 & $46.9(22.3)$ & $60.7(25.9)$ & $<0.0001$ & 180 & $44.4(21.8)$ & $57.3(24.8)$ & $<0.0001$ \\
\hline & 196 & $37.4(21.5)$ & $59.6(25.3)$ & $<0.0001$ & 182 & $36.0(20.7)$ & $53.3(24.6)$ & $<0.0001$ \\
\hline & 189 & $30.6(31.8)$ & $50.9(38.0)$ & $<0.0001$ & 179 & $33.4(31.3)$ & $48.6(33.7)$ & $<0.0001$ \\
\hline FDI & 201 & 26.9 (11.3) & $16.2(12.6)$ & $<0.0001$ & na & na & na & na \\
\hline BAP-Q5 & 122 & $15.0(5.9)$ & $10.5(6.0)$ & $<0.0001$ & na & na & na & na \\
\hline
\end{tabular}

$\dagger-p$-value based on paired t-test; BAP-Q5 - Bath Adolescent Pain Questionnaire, Painrelated worry section; FDI - Functional Disability Inventory; $\mathrm{n}$ - the total number of child and parent questionnaire pairs with valid data for both time points; $n / a$ - Not applicable as parent-proxy questionnaires are not collected; PedsQL - Pediatric Quality of Life Inventory, Generic core scales; SD - Standard deviation 
Table 6. Medications used often or daily at referral and episode end, parent report

\begin{tabular}{|l|c|c|c|c|}
\hline \multicolumn{1}{|c|}{ Drug Class } & $\begin{array}{c}\text { Number with } \\
\text { data for both } \\
\text { time points }\end{array}$ & $\begin{array}{c}\text { Using drug class } \\
\text { at referral } \\
\mathrm{n}(\%)\end{array}$ & $\begin{array}{c}\text { Using drug class } \\
\text { at episode end } \\
\mathrm{n}(\%)\end{array}$ & $\begin{array}{c}\text { p value* for } \\
\text { change }\end{array}$ \\
\hline $\begin{array}{l}\text { Paracetamol } \\
\text { (acetaminophen) }\end{array}$ & 189 & $109(57.7)$ & $68(36.0)$ & $<0.0001$ \\
\hline $\begin{array}{l}\text { Non-steroidal anti- } \\
\text { inflammatory drugs }\end{array}$ & 169 & $91(53.8)$ & $55(32.5)$ & $<0.0001$ \\
\hline $\begin{array}{l}\text { Complementary and } \\
\text { alternative medicines }\end{array}$ & 175 & $54(30.9)$ & $41(23.4)$ & 0.0533 \\
\hline $\begin{array}{l}\text { Opioids (including } \\
\text { codeine) }\end{array}$ & 162 & $31(19.1)$ & $21(13.0)$ & 0.0872 \\
\hline $\begin{array}{l}\text { Anti-neuropathic pain } \\
\text { medications }\end{array}$ & 175 & $61(34.9)$ & $66(37.7)$ & 0.5758 \\
\hline
\end{tabular}

* p-value based on McNemar's test 


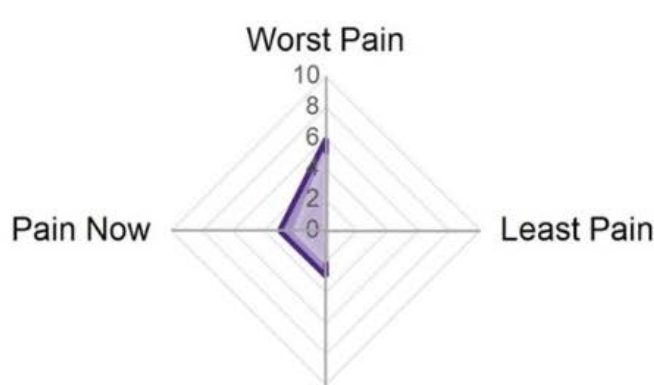

Average Pain

Referral - 24/08/2016 $\square$ Latest - 16/11/2016

PAIN SEVERITY - CHILD

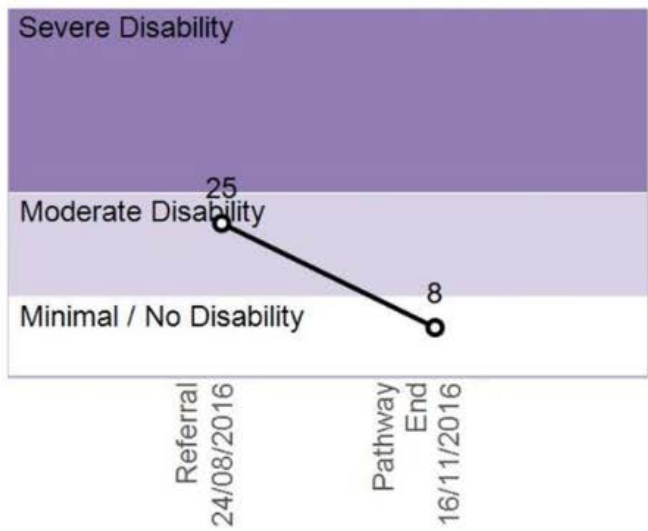

FUNCTIONAL DISABILITY INDEX

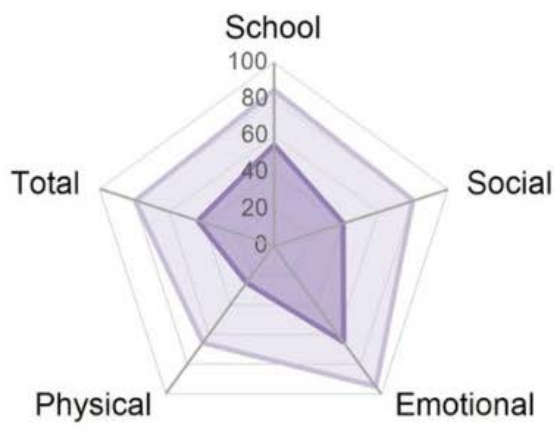

Referral - 24/08/2016 Latest - 16/11/2016

QUALITY OF LIFE - CHILD

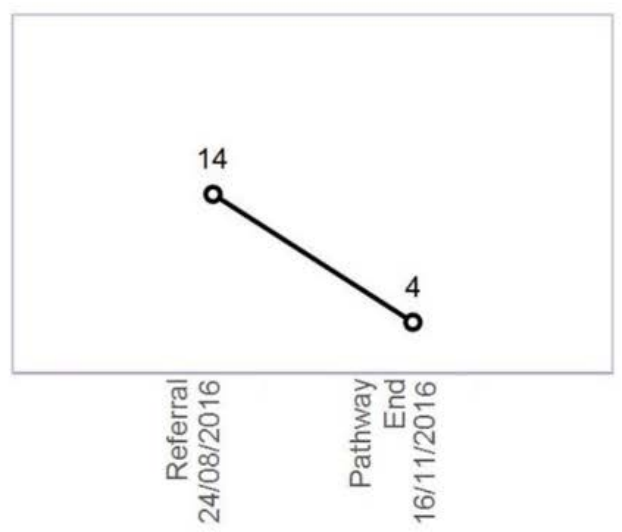

PAIN RELATED WORRIES

Figure 1 